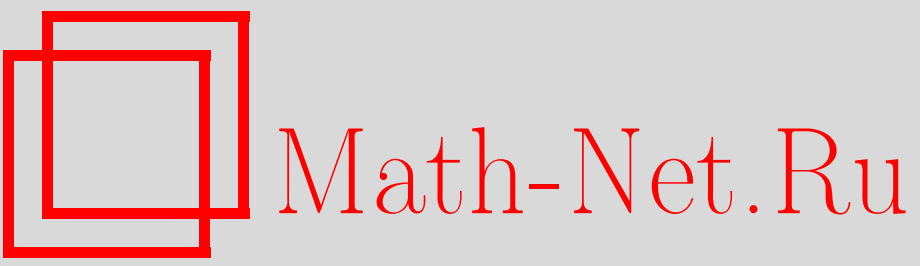

Л. В. Розовский, О вероятностях малых уклонений максимума частных сумм, Теория вероятн. и ее примен., 2009, том 54, выпуск 4, 794-801

DOI: https://doi.org/10.4213/tvp3544

Использование Общероссийского математического портала Math-Net.Ru подразумевает, что вы прочитали и согласны с пользовательским соглашением

http://www . mathnet.ru/rus/agreement

Параметры загрузки:

IP : 54.89 .56 .158

26 апреля 2023 г., $12: 44: 52$

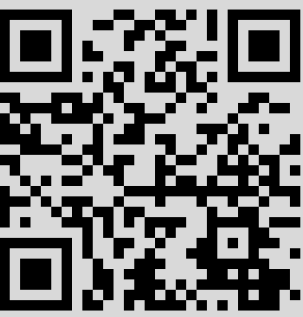




\section{О ВЕРОЯТНОСТЯХ МАЛЫХ УКЛОНЕНИЙ МАКСИМУМА ЧАСТНЫХ СУММ ${ }^{1)}$}

Пусть $S_{n}=X_{1}+\cdots+X_{n}$, где $X_{1}, X_{2}, \ldots$ - независимые копии случайной величины $X$. Предполагается, что для некоторых положительных $B_{n}$ распределение $S_{n} / B_{n}$ слабо сходится к некоторому невырожденному распределению.

Наша главная цель заключается в изучении асимптотического поведения сумм вида

$$
\sum_{n \geqslant 1} f_{n} \mathbf{P}\left(\max _{1 \leqslant k \leqslant n}\left|S_{k}\right| \leqslant r \frac{B_{n}}{\phi_{n}}\right) \quad \text { при } r \nearrow \infty
$$

где $\phi_{n} \nearrow \infty, f_{n} \geqslant 0$, а ряд $\sum f_{n}$ расходится.

Ключевые слова и фразы: малые уклонения, максимум частных сумм, размах, области притяжения, устойчивые законы.

1. Введение и результаты. Рассмотрим случайную величину $X$ и ее независимые копии $X_{1}, X_{2}, \ldots$. Положим $S_{n}=X_{1}+\cdots+X_{n}$. В дальнейшем будем предполагать, что для некоторых положительных $B_{n}$

$$
\text { распределение } B_{n}^{-1} S_{n} \text { слабо сходится к некоторому }
$$

невырожденному распределению.

Имея в виду, что это распределение обязано быть строго устойчивым с некоторым характеристическим показателем $\alpha, 0<\alpha \leqslant 2$ (см. [1], [12, (1.5)-(1.8) при $\left.a_{n}=0\right]$ ), обозначим его $F_{\alpha}$.

Наша главная цель заключается в изучении асимптотического поведения сумм вида

где

$$
\sum_{n \geqslant 1} f_{n} \mathbf{P}\left(\max _{1 \leqslant k \leqslant n}\left|S_{k}\right| \leqslant r \frac{B_{n}}{\phi_{n}}\right) \text { при } r \nearrow \infty,
$$

$$
\phi_{n} \nearrow \infty, \quad f_{n} \geqslant 0, \quad \sum_{n \geqslant 1} f_{n}=\infty .
$$

Схожие задачи рассматривались рядом авторов (см., например, [2]-[14], а также более ранние ссылки в $[16$, Дополнения к гл. VI и VII]). Потребность в подобных исследованиях изначально была стимулирована сильными предельными теоремами типа закона повторного логарифма в форме Колмогорова или Чжуна и возникает при решении ряда других задач, таких, например, как изучение числа выходов случайного блуждания за криволинейную границу.

Непосредственным поводом для настоящего исследования явилась статья [14], в которой с помощью метода сильной аппроксимации было доказано, что если $\mathbf{E} X=0$, $\mathbf{E} X^{2}=1$ и $\mathbf{E} X^{2}(\ln |X|)^{\rho / 2}<\infty$ при некотором фиксированном положительном $\rho$, то

$$
\begin{aligned}
\lim _{r \nearrow \infty} & r^{-\rho} \sum_{n \geqslant 1} \frac{(\ln n)^{\rho / 2-1}}{n} \mathbf{P}\left(\max _{1 \leqslant k \leqslant n}\left|S_{k}\right| \leqslant r \sqrt{\frac{\pi^{2} n}{8 \ln n}}\right) \\
& =\frac{4}{\pi} \Gamma\left(\frac{\rho}{2}\right) \sum_{k \geqslant 0} \frac{(-1)^{k}}{(2 k+1)^{\rho+1}} .
\end{aligned}
$$

* С.-Петербургская химико-фармацевтическая академия, кафедра высшей математики, ул. проф. Попова, 14, 197376 C.-Петербург, Россия; e-mail: 1_rozovski@mail.ru

1) Исследование выполнено при поддержке программы «Ведущие научные школы» (грант НШ-4222.2006.1) и РФФИ (грант № 06-01-00179-а). 
В настоящей статье будет показано, что утверждения более общие и более точные, нежели равенство (1.3), можно получить, используя результаты и идеи, содержащиеся в работе [1]. Так, равенство (1.3) остается справедливым без излишних моментных предположений.

Сформулируем результаты. Введем обозначения.

Определим случайную ступенчатую функцию $\xi_{n}(t), 0 \leqslant t \leqslant 1, n \geqslant 1$, положив

$$
\xi_{n}(t)=\frac{S_{k}}{B_{n}} \quad \text { при } \quad \frac{k}{n} \leqslant t<\frac{k+1}{n},
$$

где $k=0,1, \ldots, n-1, S_{0}=0$. Пусть $\{\xi(t) ; t \geqslant 0\}$ обозначает однородный процесс с независимыми приращениями такой, что $\mathbf{P}(\xi(t)<x)=F_{\alpha}\left(x t^{-1 / \alpha}\right)$ при любых положительных $t$. Мы также предполагаем, что выборочные траектории $\xi(t)$ непрерывны справа с вероятностью 1.

Обозначим через $D(0,1)$ пространство вещественных функций $f(t), 0 \leqslant t \leqslant 1$, без разрывов второго рода, непрерывных справа при $0 \leqslant t<1$ и слева при $t=1$.

Для любого борелевского множества $G \subseteq D(0,1)$ и любого положительного а положим

$$
\mathscr{P}_{G}(a)=\mathbf{P}(a \xi(\cdot) \in G), \quad \mathscr{P}_{G}^{n}(a)=\mathbf{P}\left(a \xi_{n}(\cdot) \in G\right) .
$$

Начнем со следующего довольно общего утверждения, в котором собраны обозримые и в то же время близкие к оптимальным условия, позволяющие вычислить асимптотику суммы $\sum_{n \geqslant 1} f_{n} \mathscr{P}_{G}^{n}\left(\varepsilon \phi_{n}\right)$ при $\varepsilon \searrow 0$.

Предложение 1.1. Пусть выполняются условия (1.2), а также условия

$$
\begin{aligned}
& \limsup _{n \rightarrow \infty} \frac{\phi_{n+1}}{\phi_{n}}<\infty, \\
& \limsup _{k \rightarrow \infty} \phi_{k}^{\delta} \sum_{n>k} f_{n} \phi_{n}^{-\delta}\left(\sum_{n=1}^{k} f_{n}\right)^{-1}<\infty \quad(\exists \delta>0) .
\end{aligned}
$$

Предположим, что борелевское множество $G \subseteq D(0,1)$ таково, что

$$
\begin{aligned}
& \sup _{a>0}\left|\mathscr{P}_{G}^{n}(a)-\mathscr{P}_{G}(a)\right| \rightarrow 0, \quad n \rightarrow \infty, \\
& \inf _{0<b<a} \mathscr{P}_{G}(b)>0 \quad(\forall a \in(0, \infty))
\end{aligned}
$$

$u$, кроме того, при некотором $\gamma>\delta$ (где $\delta$ определяется условием (1.7))

$$
\begin{gathered}
\sup _{a>0} a^{\gamma} \mathscr{P}_{G}(a)<\infty, \\
\limsup _{n \rightarrow \infty} \sup _{1<a<\phi_{n}} a^{\gamma} \mathscr{P}_{G}^{n}(a)<\infty .
\end{gathered}
$$

Тогда

$$
\sum_{n \geqslant 1} f_{n} \mathscr{P}_{G}^{n}\left(\varepsilon \phi_{n}\right) \sim \sum_{n \geqslant 1} f_{n} \mathscr{P}_{G}\left(\varepsilon \phi_{n}\right), \quad \varepsilon \searrow 0 .
$$

Теперь обсудим условия предложения 1.

3 а м е ч а н и е 1. Если

$$
0 \leqslant f_{n} \sim \int_{\phi_{n}}^{\phi_{n+1}} g(u) \frac{d u}{u}, \quad n \rightarrow \infty,
$$

где положительная функция $g(u)$ удовлетворяет условиям

$$
\begin{gathered}
\int_{1}^{\infty} g(u) \frac{d u}{u}=\infty, \\
\frac{g(u)}{u^{\delta}} \text { убывает при некотором } \delta>0 \text { и всех } \\
\text { достаточно больших } u,
\end{gathered}
$$


то имеет место (1.7). Кроме того, если

$$
\phi_{n} \nearrow \infty, \quad \phi_{n+1} \sim \phi_{n}, \quad n \rightarrow \infty,
$$

$$
\mathscr{P}_{G}(a) \text { - невозрастающая положительная функция } a,
$$

TO

$$
\sum_{n \geqslant 1} f_{n} \mathscr{P}_{G}\left(\varepsilon \phi_{n}\right) \sim \psi_{G}(\varepsilon)=\int_{1}^{\infty} g(u) \mathscr{P}_{G}(\varepsilon u) \frac{d u}{u}, \quad \varepsilon \searrow 0 .
$$

3 а м е ч а н и е 2. Пусть положительная функция $g(u)$ правильно меняется на бесконечности с показателем $\rho \geqslant 0$. Тогда из условий (1.16) и (1.10а) следует, что при $\varepsilon \searrow 0$

$$
\psi_{G}(\varepsilon) \sim g\left(\frac{1}{\varepsilon}\right) \int_{0}^{\infty} u^{\rho-1} \mathscr{P}_{G}(u) d u, \quad \rho>0,
$$

а если имеет место (1.13), то

$$
\psi_{G}(\varepsilon) \sim \mathscr{P}_{G}(0) \int_{1}^{1 / \varepsilon} g(u) \frac{d u}{u}, \quad \rho=0,
$$

где

$$
\mathscr{P}_{G}(0)=\lim _{a \searrow 0} \mathscr{P}_{G}(a) .
$$

Отметим (см. [12, замечание 2$]$ ), что если $f_{\gamma}(u)$ правильно меняется на бесконечности с показателем $\gamma>-1$ и $g(u)=f_{\gamma}(\ln u)$, то

$$
\int_{1}^{z} g(u) \frac{d u}{u} \sim \frac{\ln z}{1+\gamma} g(z), \quad z \rightarrow \infty
$$

если $g(u)=f_{\gamma}(\ln \ln u) / \ln u$, то

$$
\int_{1}^{z} g(u) \frac{d u}{u} \sim \frac{\ln z \ln \ln z}{1+\gamma} g(z), \quad z \rightarrow \infty
$$

Заметим также, что в некоторых случаях коэффициент $\int_{0}^{\infty} u^{\rho-1} \mathscr{P}_{G}(u) d u(\rho>0)$ может быть вычислен.

Для положительного $a$ и борелевского множества $G \subseteq D(0,1)$ положим

$$
a G=\left\{f \in D(0,1): a^{-1} f(t) \in G\right\} .
$$

3 а м е ч а н и е 3 . Пусть

$$
a G \subseteq b G \quad(\forall 0<a<b<\infty) .
$$

Тогда из условия (1.10a), а также из условий

$$
\begin{gathered}
\mathscr{P}_{G}(a)-\text { непрерывная положительная функция } a, \\
\mathscr{P}_{\partial G}(a)=0(\forall a>0), \text { где } \partial G-\text { граница } G,
\end{gathered}
$$

и (см. (1.20))

$$
\limsup _{n \rightarrow \infty} \lim _{a \searrow 0} \mathscr{P}_{G}^{n}(a) \leqslant \mathscr{P}_{G}(0)
$$

следует (1.8). Кроме того, из (1.21) и (1.22) вытекает условие (1.9).

Введем класс $\mathscr{N}$ множеств

$$
G=G\left(c ; t_{1}, t_{2}\right)=\left\{f \in D(0,1): f(0)=0,|f(t)|<c ; t_{1} \leqslant t<t_{2}\right\},
$$

где $0<c<\infty, 0 \leqslant t_{1}<t_{2} \leqslant 1$.

3 а м е ч ан и е 4 . Пусть

$$
\limsup _{n \rightarrow \infty} \frac{\ln \phi_{n}}{n}=0 .
$$


Если

$$
G \subseteq \bigcup_{1 \leqslant i \leqslant N} G_{i}
$$

при некоторых $G_{i} \in \mathscr{N}$ и конечном $N=1,2, \ldots$, то условия (1.10) выполняются при любом $\gamma>0$.

Отметим, что (1.26) является следствием (1.15).

3 а м е ч а н и е 5 . Пусть

$$
0<F_{\alpha}(0)<1
$$

Если

$$
G \supseteq \widetilde{G} \quad(\exists \widetilde{G} \in \mathscr{N})
$$

то справедливо (1.9).

Заметим, что предположение (1.28) информативно лишь при $0<\alpha<1$.

Обращаем внимание на то, что множества $G$ типа

$$
\begin{aligned}
& J_{a}=\left\{f \in D(0,1): f(0)=0, \sup _{0 \leqslant t \leqslant 1} f(t)-\inf _{0 \leqslant t \leqslant 1} f(t) \leqslant a\right\}, \quad a>0, \\
& I_{c}^{b}=\left\{f \in D(0,1): f(0)=0, c \leqslant \inf _{0 \leqslant t \leqslant 1} f(t) \leqslant \sup _{0 \leqslant t \leqslant 1} f(t) \leqslant b\right\}, \quad c<0<b,
\end{aligned}
$$

удовлетворяют условиям (1.27), (1.29) при $N=1$.

Далее приведем некоторые следствия предложения 1.

Для краткости обозначим $\Delta \phi_{n}=\phi_{n+1}-\phi_{n}$,

$$
m_{n}=\min _{0 \leqslant k \leqslant n} S_{k}, \quad M_{n}=\max _{0 \leqslant k \leqslant n} S_{k}, \quad R_{n}=M_{n}-m_{n} .
$$

Теорема 1.1. Пусть удовлетворяются условия (1.15), (1.28), а положительная функиия $g(u)$ медленно меняется на бесконечности с показателем $\rho \geqslant 0$ и при $\rho=0$ удовлетворяет условию (1.13). Тогда

1) для любих $0<a, b<\infty$

$$
\begin{gathered}
\lim _{r \nearrow \infty}\left(\int_{1}^{r} g(u) \frac{d u}{u}\right)^{-1} \sum_{n \geqslant 1} g\left(\phi_{n}\right) \frac{\Delta \phi_{n}}{\phi_{n}} \mathbf{P}\left(-a r \frac{B_{n}}{\phi_{n}} \leqslant m_{n} \leqslant M_{n} \leqslant b r \frac{B_{n}}{\phi_{n}}\right) \\
=A_{\alpha}(\rho ; a, b)
\end{gathered}
$$

2) справедливо равенство

$$
\lim _{r \nearrow \infty}\left(\int_{1}^{r} g(u) \frac{d u}{u}\right)^{-1} \sum_{n \geqslant 1} g\left(\phi_{n}\right) \frac{\Delta \phi_{n}}{\phi_{n}} \mathbf{P}\left(R_{n} \leqslant r \frac{B_{n}}{\phi_{n}}\right)=C_{\alpha}(\rho) .
$$

Здесь постоянные $A_{\alpha}(\rho ; a, b)$ и $C_{\alpha}(\rho)$ в случае положительного $\rho$ равны $\rho \int_{0}^{\infty} u^{\rho-1} \mathscr{P}_{G}(u) d u$ при $G=I_{-a}^{b}$ и $G=J_{1}$ соответственно, а в случае $\rho=0$ равны единице.

Кроме того, при $\rho>0$

$$
\int_{1}^{r} g(u) \frac{d u}{u} \sim \frac{g(r)}{\rho}, \quad r \rightarrow \infty
$$

3 а м е чани е 6.1 1) $A_{\alpha}(\rho ; 1,1)=\rho^{-1} \mathbf{E} \Xi^{-\rho}$ и $C_{\alpha}(\rho)=\rho^{-1} \mathbf{E} R^{-\rho}$, где $\Xi=$ $\sup _{0 \leqslant t \leqslant 1}|\xi(t)|, R=\sup _{0 \leqslant t \leqslant 1} \xi(t)-\inf _{0 \leqslant t \leqslant 1} \xi(t)$.

2) Пусть $\rho>0$. Если $\alpha=2$, т.е. $\xi(\cdot)$ - стандартный винеровский процесс $W(\cdot)$, то

$$
A_{2}(\rho ; a, b)=\frac{2 \rho}{\pi}\left(\frac{c \sqrt{2}}{\pi}\right)^{\rho} \Gamma\left(\frac{\rho}{2}\right) \sum_{k \geqslant 0}(2 k+1)^{-1-\rho}
$$




$$
\begin{gathered}
\times \sin (2 k+1) \pi \frac{a}{c}, \quad c=a+b, \\
C_{2}(\rho)=\frac{4(1+\rho)}{\pi^{2}}\left(\frac{\sqrt{2}}{\pi}\right)^{\rho} \Gamma\left(\frac{\rho}{2}\right) \sum_{k \geqslant 0}(2 k+1)^{-(2+\rho)} .
\end{gathered}
$$

Чтобы получить (1.3), воспользуемся первым утверждением теоремы 1 при

$$
\begin{gathered}
\alpha=2, \quad \mathbf{E} X=0, \quad \mathbf{E} X^{2}=1, \quad g(u)=u^{\rho}, \quad \phi_{n}=\sqrt{\frac{8 \ln n}{\pi^{2}}}, \\
a=b=1, \quad B_{n}=\sqrt{n},
\end{gathered}
$$

и тем, что

$$
A_{2}(\rho, 1,1)=\frac{2 \rho}{\pi}\left(\frac{2 \sqrt{2}}{\pi}\right)^{\rho} \Gamma\left(\frac{\rho}{2}\right) \sum_{k \geqslant 0}(-1)^{k}(2 k+1)^{-1-\rho} .
$$

2. Доказательства. Докажем предложение 1. Пусть положительная функция $M=M(\varepsilon)$ растет к бесконечности при $\varepsilon \searrow 0$. Обозначим $n_{1}=\max \left(n: \phi_{n} \leqslant 1 / \varepsilon\right)$, $n_{2}=\max \left(n: \phi_{n} \leqslant M / \varepsilon\right)$ и

$$
\left(\sum_{1 \leqslant n<M}+\sum_{M \leqslant n \leqslant n_{2}}+\sum_{n>n_{2}}\right) f_{n} \mathscr{P}_{G}^{n}\left(\varepsilon \phi_{n}\right)=S_{1}+S_{2}+S_{3} .
$$

Из (1.8), (1.9) следует, что если функция $M$ достаточно медленно растет к бесконечности, то

$$
\mathscr{P}_{G}^{n}\left(\varepsilon \phi_{n}\right) \sim \mathscr{P}_{G}\left(\varepsilon \phi_{n}\right), \quad \varepsilon \searrow 0,
$$

равномерно по $n$ таким, что $M \leqslant n \leqslant n_{2}$, и поэтому

$$
S_{2} \sim \sum_{M \leqslant n \leqslant n_{2}} f_{n} \mathscr{P}_{G}\left(\varepsilon \phi_{n}\right), \quad \varepsilon \searrow 0 .
$$

Принимая во внимание (см. (1.2) и (1.9)), что

$$
S(\varepsilon)=\sum_{n \geqslant 1} f_{n} \mathscr{P}_{G}\left(\varepsilon \phi_{n}\right) \rightarrow \infty, \quad \varepsilon \searrow 0,
$$

получим

$$
\sum_{1 \leqslant n<M} f_{n}\left(\mathscr{P}_{G}^{n}\left(\varepsilon \phi_{n}\right)+\mathscr{P}_{G}\left(\varepsilon \phi_{n}\right)\right) \leqslant 2 \sum_{1 \leqslant n<M} f_{n}=o(S(\varepsilon)), \quad \varepsilon \searrow 0 .
$$

Из условий (1.10) и (1.7) следует, что при некоторых положительных $\gamma, \delta$

$$
\begin{aligned}
& \sum_{n>n_{2}} f_{n}\left(\mathscr{P}_{G}^{n}\left(\varepsilon \phi_{n}\right)+\mathscr{P}_{G}\left(\varepsilon \phi_{n}\right)\right) \leqslant A_{1} M^{-\gamma} \sum_{n>n_{1}} f_{n}\left(\varepsilon \phi_{n}\right)^{-\delta} \\
& \leqslant A_{2} M^{-\gamma}\left(\varepsilon \phi_{n_{1}}\right)^{-\delta} \sum_{n=1}^{n_{1}} f_{n} \leqslant A_{2} M^{-\gamma} \frac{\left(\phi_{n_{1}+1} / \phi_{n_{1}}\right)^{\delta}}{\inf _{0<b \leqslant 1} \mathscr{P}_{G}(b)} S(\varepsilon),
\end{aligned}
$$

где постоянные $A_{i}$ не зависят от $\varepsilon$. Таким образом, из (1.6), (1.9), (2.1)-(2.3) вытекает (1.11). Тем самым, доказательство предложения 1 закончено.

Теперь займемся замечаниями.

Сначала проверим справедливость замечания 1. То, что (1.12), (1.14) влечет (1.7), было доказано в [12, доказательство следствия 2]. Покажем, что (1.17) следует из (1.12)-(1.16). В силу условия (1.16) имеем

$$
\mathscr{P}_{G}\left(\varepsilon \phi_{n}\right) \int_{\phi_{n}}^{\phi_{n+1}} g(u) \frac{d u}{u} \geqslant \int_{\phi_{n}}^{\phi_{n+1}} \mathscr{P}_{G}(\varepsilon u) g(u) \frac{d u}{u},
$$

и при $0<\lambda<\phi_{n} / \phi_{n+1} \leqslant 1$ выполнено неравенство

$$
\mathscr{P}_{G}\left(\varepsilon \phi_{n}\right) \int_{\phi_{n}}^{\phi_{n+1}} g(u) \frac{d u}{u} \leqslant \int_{\phi_{n}}^{\phi_{n+1}} \mathscr{P}_{G}(\varepsilon \lambda u) g(u) \frac{d u}{u} .
$$


Кроме того, легко убедиться в том, что из (1.14), (1.16) следует

$$
\psi_{G}(\varepsilon) \leqslant \psi_{G}(\lambda \varepsilon) \leqslant \lambda^{-\delta} \psi_{G}(\varepsilon)+g(1)\left(\lambda^{-\delta}-1\right) \delta^{-1}, \quad 0<\lambda<1 .
$$

Теперь, с учетом (1.12), соотношение (1.17) представляется достаточно очевидным.

Доказательство справедливости замечания 2 может быть проведено аналогично доказательству замечания 2 (утверждения 1) и 2)) в [12] (см. также [13, формулы (3.2)-(3.5)]).

Замечание 3 проверяется с помощью стандартных рассуждений - как в $[16$, п. 5, теорема 13], с учетом того, что $\lim _{n \rightarrow \infty} \mathscr{P}_{G}^{n}(a)=\mathscr{P}_{G}(a)(\forall a>0)$ в соответствии с (1.1) и хорошо известными теоремами о сходимости вероятностных процессов [18], [19].

Докажем теперь замечание 4. Изучая (1.10b), мы можем ограничиться множеством $G \in \mathscr{N}$. Обозначим (см. $(1.25)) m=\left[n\left(t_{2}-t_{1}\right)\right], k_{0}=\left[n t_{1}\right], \delta=2 c B_{n} / a$. Тогда по (1.4) и $[1$, формула $(32)]$ (см. обозначения в $(1.30)$ )

$$
\begin{aligned}
\mathscr{P}_{G}^{n}(a) & \leqslant \mathbf{P}\left(\max _{k_{0} \leqslant i \leqslant m+k_{0}}\left|S_{i}\right|<\frac{c B_{n}}{a}\right) \\
& \leqslant \mathbf{P}\left(\max _{0 \leqslant j \leqslant m}\left|S_{j}\right|<\delta\right) \leqslant\left[\mathbf{P}\left(R_{k}<2 \delta\right)\right]^{l} \leqslant\left[\mathbf{P}\left(\max _{0 \leqslant i \leqslant k}\left|S_{i}\right|<2 \delta\right)\right]^{l}
\end{aligned}
$$

для любого $1 \leqslant k \leqslant m$ и для $l=[m / k]$.

Пусть $a<\phi_{n}$ достаточно велико. Положим в $(2.4) k=[\mathrm{m} / \ln a]$. Принимая во внимание (1.26) и то, что последовательность $B_{n}$ правильно меняется, находим, что вероятность $\mathbf{P}\left(\max _{0 \leqslant i \leqslant k}\left|S_{i}\right|<2 \delta\right)$ может быть сделана произвольно малой, и легко выводим (1.10b) из (2.4). Условие (1.10a) можно получить аналогично с упрощениями.

Справедливость замечания 5 следует непосредственно из [1, теорема 1].

Пусть положительная функция $g(u)$ правильно меняется на бесконечности с показателем $\rho \geqslant 0$. Положим $f_{n}=g\left(\phi_{n}\right) \Delta \phi_{n} / \phi_{n}$. Очевидно (см. обозначения после замечания 5), что вероятности $\mathbf{P}\left(-a r B_{n} / \phi_{n} \leqslant m_{n} \leqslant M_{n} \leqslant b r B_{n} / \phi_{n}\right)$ и $\mathbf{P}\left(R_{n} \leqslant r B_{n} / \phi_{n}\right)$ совпадают с $\mathscr{P}_{G}\left(\phi_{n} / r\right)$ при $G=I_{-a}^{b}$ и $G=J_{1}$ соответственно. Поэтому, чтобы доказать теоремы 1 и 2 , воспользуемся предложением 1 . Нам требуется проверить выполнение условий (1.2), (1.6)-(1.10).

Пусть условия теоремы 1, т.е. (1.15), (1.28) и (1.13), выполняются (заметим, что (1.13) в случае положительного $\rho$ имеет место). Без потери общности считая выполненным условие (1.14), с помощью замечания 1 получим (1.2), (1.6) и (1.7). Условия (1.9), (1.10) и (1.8) справедливы в силу замечаний 4 и 5 соответственно (условие (1.21) при $G=I_{-a}^{b}$ или $G=J_{1}$, очевидно, выполняется, причем $\left.\mathscr{P}_{G}(0)=1\right)$.

Итак (см. (1.11)), нами показано, что при $G=I_{-a}^{b}$ или $G=J_{1}$

$$
\sum_{n \geqslant 1} f_{n} \mathscr{P}_{G}^{n}\left(\frac{\phi_{n}}{r}\right) \sim \sum_{n \geqslant 1} f_{n} \mathscr{P}_{G}\left(\frac{\phi_{n}}{r}\right), \quad r \nearrow \infty .
$$

Для завершения доказательства теоремы 1 остается оценить сумму справа, используя соотношения (1.17)-(1.19).

Заметим, что прямое применение найденной в [1] логарифмической асимптотики вероятностей вида $\mathscr{P}_{G}\left(\phi_{n} / r\right)$ для исследования поведения соответствующих рядов в теореме 1 невозможно, поскольку для этой цели требуются оценки, равномерные по параметру $r$.

Соотношения (1.31), (1.32) следуют из предложения 1 и соответствующих представлений вероятностей $G(a, b)=\mathbf{P}(-a<W(t)<b ; 0 \leqslant t \leqslant 1)$ и $\mathbf{P}(R<r)$, где $R=\sup _{0 \leqslant t \leqslant 1} W(t)-\inf _{0 \leqslant t \leqslant 1} W(t)$.

Так, чтобы проверить (1.31), нами применяется равенство (см. [17])

$$
G(a, b)=\frac{4}{\pi} \sum_{j \geqslant 0} \frac{1}{2 j+1} e^{-((2 j+1) \pi)^{2} /\left(2 c^{2}\right)} \sin \frac{(2 j+1) \pi a}{c}
$$


где $a, b$ положительны и $c=a+b$.

Чтобы получить (1.32), мы используем следующее, принадлежащее Феллеру (см. [15]), представление плотности

$$
\frac{d \mathbf{P}(R<r)}{d r}=\delta(r)=\sqrt{\frac{2}{\pi}} r^{-1} L^{\prime}\left(\frac{r}{2}\right),
$$

где

$$
L(z)=\sqrt{2 \pi} z^{-1} \sum_{k \geqslant 1} e^{-(2 k-1)^{2} \pi^{2} /\left(8 z^{2}\right)} .
$$

Мы должны показать, что (см. (1.5), (1.32) и обозначения после замечания 5)

$$
I=\int_{0}^{\infty} u^{\rho-1} \mathscr{P}_{J_{1}}(u) d u=C_{2}(\rho) .
$$

Действительно,

$$
\begin{aligned}
I & =\int_{0}^{\infty} \mathbf{P}\left(R<\frac{1}{u}\right) d u^{\rho}=\int_{0}^{\infty} u^{\rho-2} \delta\left(\frac{1}{u}\right) d u=\sqrt{\frac{2}{\pi}} \int_{0}^{\infty} u^{\rho-1} L^{\prime}\left(\frac{1}{2 u}\right) d u \\
& =2 \sqrt{\frac{2}{\pi}} \int_{0}^{\infty} u^{\rho+1}\left(-d L\left(\frac{1}{2 u}\right)\right)=2 \sqrt{\frac{2}{\pi}}(1+\rho) \int_{0}^{\infty} u^{\rho} L\left(\frac{1}{2 u}\right) d u .
\end{aligned}
$$

Чтобы получить нужный результат, остается использовать здесь соотношение (2.5).

\section{СПИСОК ЛИТЕРАТУРЫ}

1. Могульский A. A. Малые уклонения в пространстве траекторий. - Теория вероятн. и ее примен., 1974, т. 19, в. 4, с. $755-765$.

2. Chen R. A remark on the tail probability of a distribution. - J. Multivariate Anal., 1978 , v. 8, № 2, p. 328-333.

3. Гафуров М.У., Сластников А.Д. О распределении момента последнего выхода, эксцесса и числа выходов случайного блуждания за криволинейную границу. Докл. АН СССР, 1981, т. 257, № 3, с. 526-529.

4. Гафуров М. У., Ротарь В.И. О выходе случайного блуждания за криволинейную границу. - Теория вероятн. и ее примен., 1983, т. 28, в. 1, с. 169-175.

5. Розовский Л.В. Некоторые оценки для вероятностей односторонних больших уклонений. - Теория вероятн. и ее примен., 1985, т. 30, в. 4, с. 800-804.

6. Розовский Л. В. Нормальная аппроксимация при вычислении скорости сходимости в слабом законе больших чисел. - Матем. заметки, 1986, т. 40, № 2, с. 252-268.

7. Li D. L., Wang X. C., Rao M. B. Some results on convergence rates for probabilities of moderate deviations for sums of random variables. - Internat. J. Math. Math. Sci., 1992, v. 15, № 3, p. 481-497.

8. Spătaru A. Precise asymptotics in Spitzer's law of large numbers. - J. Theoret. Probab., 1999, v. 12, № 3, p. 811-819.

9. Gut A., Spătaru A. Precise asymptotics in the Baum-Katz and Davis laws of large numbers. - J. Math. Anal. Appl., 2000, v. 248, № 1, p. 233-246.

10. Gut A., Spătaru A. Precise asymptotics in the law of the iterated logarithm. - Ann. Probab., 2000, v. 28, № 4, p. 1870-1883.

11. Zhang L. X. On the rates of the other law of the logarithm. arXiv: math/0610521.

12. Розовский Л. В. О точной асимптотике в слабом законе больших чисел для сумм независимых случайных величин с общей функцией распределения из области притяжения устойчивого закона. - Теория вероятн. и ее примен., 2003, т. 48, в. 3 , с. $589-596$.

13. Розовский Л. В. О точной асимптотике в слабом законе больших чисел для сумм независимых случайных величин с общей функцией распределения из области притяжения устойчивого закона. II. - Теория вероятн. и ее примен., 2004, т. 49, B. 4 , с. $803-813$. 
14. Pang T., Lin Z. On the rates of the Chung-type law of logarithm. - Теория вероятн. и ее примен., 2009, т. 54 , в. 4 , с. $814-827$.

15. Feller $W$. The asymptotic distribution of the range of sums of independent random variables. - Ann. Math. Statist., 1951, v. 22, № 3, p. 427-432.

16. Петров В.В. Предельные теоремы для сумм независимых случайных величин. М.: Наука, 1987, 317 с.

17. Феллер В. Введение в теорию вероятностей и ее приложения, т. 2. М.: Мир, 1984, $752 \mathrm{c}$.

18. Прохоров Ю. В. Сходимость случайных процессов и предельные теоремы теории вероятностей. - Теория вероятн. и ее примен., 1956, т. 1, в. 2, с. 177-238.

19. Скороход A.B. Случайные процессы с независимыми приращениями. М.: 1986, $320 \mathrm{c}$.

Поступила в редакцию 15.VII.2007

Исправленный вариант

03.VI.2008

(c) 2009 г.

ТЮРИН И. С.*

\title{
РАВНОМЕРНЫЕ ЗАКОНЫ СРЕДНИХ ДЛЯ ПОСЛЕДОВАТЕЛЬНОСТЕЙ ЗАВИСИМЫХ СЛУЧАЙНЫХ ЭЛЕМЕНТОВ
}

\begin{abstract}
Изучаются классы последовательностей, обладающих свойствами зависимости, обобщающими абсолютную регулярность и перемешивание по Ибрагимову. Техника каплинга позволяет установить по отношению к ним равномерные законы средних для тех же классов функций, что и в случае, когда рассматриваемая последовательность состоит из независимых случайных элементов.
\end{abstract}

Ключевые слова и фразы: равномерная сходимость, закон больших чисел, слабая зависимость, каплинг.

1. Введение. Говорят, что для класса функций $\mathscr{F}$ выполнен равномерный закон средних (РЗС) по отношению к последовательности случайных элементов $\left(X_{j}\right)_{j \geqslant 1}$, если

$$
\lim _{n \rightarrow \infty} \sup _{f \in \mathscr{F}} \frac{1}{n}\left|\sum_{k=1}^{n} f\left(X_{k}\right)-\mathbf{E} f\left(X_{k}\right)\right|=0 \quad \text { п.н. }
$$

В этом случае соответствующий класс функций называют классом ГливенкоКантелли.

Предельные теоремы такого типа обобщают законы больших чисел и являются ключевыми для теории статистического обучения, проблем распознавания образов, восстановления зависимостей по эмпирическим данным (см. [1]). Равномерные законы средних по отношению к последовательностям независимых одинаково распределенных случайных элементов хорошо изучены. В [2] найдены необходимые и достаточные условия выполнения (1) для равномерно ограниченного класса функций (т.е. $|f(x)| \leqslant K$ при всех $f, x$ и некоторой константе $K)$. В работах М. Талаграна [3] и [4] детально изучены классы Гливенко-Кантелли.

* Московский государственный университет им. М. В. Ломоносова, механико-математический факультет, кафедра теории вероятностей, Ленинские горы, 119991 Москва, Россия; e-mail: itiurin@gmail.com 